\title{
SCALING RAINFALL SERIES WITH A MULTIFRACTAL MODEL
}

\author{
Assela PATHIRANA ${ }^{1}$, Srikantha HEARTH ${ }^{2}$, Katumi MUSIAKE ${ }^{3}$ \\ ${ }^{1}$ Student member of JSCE, M. Eng., Institute of Industrial Science, University of Tokyo (4-6-1, Komaba, \\ Meguro-Ku, Tokyo 153-8505, JAPAN) \\ ${ }^{2}$ Member of JSCE, Dr. of Eng., Guest Professor, Institute of Industrial Science, University of Tokyo \\ (4-6-1, Komaba, Meguro-Ku, Tokyo 153-8505, JAPAN) \\ ${ }^{3}$ Fellow of JSCE, Dr. of Eng., Professor, Institute of Industrial Science, University of Tokyo (4-6-1, \\ Komaba, Meguro-Ku, Tokyo 153-8505, JAPAN)
}

\begin{abstract}
A multifractal model is used to derive the hourly rainfall distributions from those observed at daily scale for a number of rainfall series observed near Tokyo. With these distributions a cascading model is used to derive a multifractal fields which has similar scaling properties as the original rainfall series. Synthetic hourly rainfall series are generated based on these multifractal field. The results are verified with numerous comparisons with original hourly rainfall distributions. It is proved that the adopted method can produce an accurate representation of hourly rainfall distributions using only daily rainfall as source data.
\end{abstract}

Key Words: Synthetic Rainfall, Multifractals, Scaling, Multiplicative cascade, Stochastic model

\section{INTRODUCTION}

Rainfall data, of hourly or higher resolutions are required for many real world water resources engineering problems, related to diverse fields: urban storm drainage, soil erosion and response studies of small watersheds to name a few. Highresolution data acquisition has been an expensive task; at least until recently and hence, repositories of such data are severely limited both in number and available duration in many parts of the world at the present day. However, daily rainfall series of considerable length are widely available for many locations, including those of developing countries. This situation has made it beneficial to device means to estimate high-resolution rainfall distributions from those observed at a much lower resolution.

Except for a several ad-hoc methods related to stochastic models (Pathirana, et al. ${ }^{1 \text { ) }}$ ) and using exponential distribution within a day $\left(\right.$ Tanimoto ${ }^{2)}$ ), almost all of the scaling models proposed in recent literature use scaling theories based on scualing described by fractals and multifractals. (Lovejoy and Schertzer ${ }^{3)}$, Olsson $^{4)}$,Svensson et al. ${ }^{5)}$ ) Since the fractal based models adopts the discontinuity in distributions into the theory (rather than as exceptions as practiced in traditional continu- ous mathematics), they are strongly favoured to model rainfall, which by nature is discontinuous, to some degree in spatial domain and to a much larger degree in the temporal domain.

While there are numerous attempts to establish rainfall as a multi-scaling process only a few reported cases exist, where the specific problem of deriving the distribution at hourly scale using observations made at daily level was attempted $\left(\right.$ Ngugen and Pandey $\left.{ }^{6}\right)$ ). However, the practical significance of such studies are limited, from the viewpoint of the application in the real-world problems, unless the complete cycle of analysis and modelling of (daily) rainfall as a multifractal process, establishing hourly intensity distributions, generation of synthetic (hourly) rainfall series and verification with actual observations made at hourly scale. Not only the distributions of intensities and standard time series properties must match but also the properties specific to rainfall series, such as distribution of wet and dry periods at various scales should be agreeing.

This paper covers all the above aspects. While the reader is referred to (pathirana, et.al. ${ }^{7)}$ ) for detailed process of multifractal analysis and modelling of rainfall time series, sufficient details on the methodology have been given in the following sections. The prime focus of this article is on the 
generation of synthetic rainfall time series and the comparison of those with the real observations.

\section{THEORY}

The intensity distribution of a multifractal field can be expressed as,

$$
P\left(\phi_{\lambda} \geq \lambda^{\gamma}\right) \sim \lambda^{-c(\gamma)}
$$

where $\lambda$ is the non-dimensional scale obtained by dividing the largest scale of interest $T$ by the scale $t . \phi$ is the normalized field. $\gamma$ is a scaling exponent and $c(\gamma)$ is known as the codimension function which takes the following form for fields generated by a multiplicative cascade process (see Fig. 1) (Tessier, et al. ${ }^{8)}$ )

$$
\begin{aligned}
& c(\gamma)= \begin{cases}C_{1}\left(\frac{\gamma}{C_{1} \alpha^{\prime}}+\frac{1}{\alpha}\right)^{\alpha^{\prime}} & \alpha \neq 1 \\
C_{1} \exp \left(\frac{\gamma}{C_{1}}-1\right) & \alpha=1\end{cases} \\
& \text { (for } 0 \leq \alpha \leq 2)
\end{aligned}
$$

where $1 / \alpha+1 / \alpha^{\prime}=1 . C_{1}$ and $\alpha$ are multifractal model parameters. Fields with $0<\alpha<2$ are generated by Lèvy-stable probability distributions.

\section{ANALYSIS OF THE OBSERVED RAINFALL}

The following method has proven to give better results with rainfall series with practically available precisions and durations (pathirana, et.al. ${ }^{7)}$ ): First the eqation 1 is rearranged in the following form by introducing a constant $b$.

$$
c(\gamma)=-\frac{\log \left(P\left(\phi_{\lambda} \geq \lambda^{\gamma}\right) / b\right)}{\log (\lambda)}
$$

Once the value of the constant $b$ is determined (see pathirana, et al. ${ }^{7}$ ) for details), this equation makes it possible to calculate an estimate for the $c(\gamma)$ function at each resolution. The ultimate value for $c(\gamma)$ is determined as the mean of all available estimates.

Rainfall time series from 17 gauging stations near Tokyo area (see Fig. 2) were selected for the analysis. Hourly rainfall was accumulated to obtain daily series. It was found that the scaling regime extends from $1 \mathrm{hr}$ scale to only $48 \mathrm{~h}$ scale, which makes only the $24 \mathrm{~h}$ and $48 \mathrm{~h}$ estimates of $c(\gamma)$ available. Table 1 shows the multifractal parameters estimated for those rainfall series, using $24 \mathrm{~h}$ and $48 \mathrm{~h}$ estimates of $c(\gamma)$. Reader is referred to pathirana, et.al. ${ }^{7}$ for details on the scaling regime and for the verification of the multifractal model at this stage.

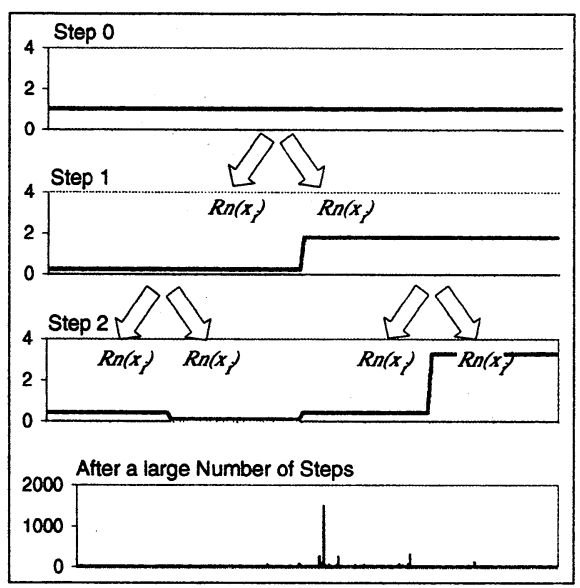

Fig. 1 A Multiplicative Cascade Process generates a multifractal field. $R n(x)$ is a function of a random variable based on a specific probability distribution that affects the distribution of values in the final multifractal field.

\section{MULTIFRACTAL SIMULATION MODEL}

The cascade process shown in Fig. 1 is the basis of a number of fractals and multifractal distribution models: Novikov and Stewart ${ }^{9)}$, Yaglom ${ }^{10)}$, Mandelbrot $^{11)}$, Frisch et al. ${ }^{12)}$, Benzi et al. ${ }^{13)}$, Meneveau and Sreenivasaan ${ }^{14}$ to name a few. In case of universal multifractals the function of a random variable $R n(x)$ must take the form: $R_{n}(x)=\operatorname{Exp}(A x+B)$, where $x$ is a Lèvy-stable random variable. $A$ and $B$ are constants. A Lèvystable random variable takes the form

$$
\begin{aligned}
& P(X>x) \sim|x|^{-\alpha} \quad(0<x) \\
& P(X>x) \sim \exp \left(-|x|^{\alpha^{\prime}}\right) \quad(x<0) \\
& (\alpha<2) \text { and } 1 / \alpha+1 / \alpha^{\prime}=1
\end{aligned}
$$

Gupta and Waymire ${ }^{15)}$, Schertzer and Lovejoy $^{16)}$,Brax and Peshanski ${ }^{17)}$, among others, have produced cascade models based on Lèvystable distribution.

While it is difficult to provide a close-form solution for the distribution (with the exception of $\alpha=2$ case where the distribution is log-normal), Lèvy-stable random variables can be generated numerically. See Wilson et al. ${ }^{18)}$ and Grigoriu ${ }^{19)}$ for a numerical algorithm to generate Lèvy-stable random variables.

The multifractal simulation model used in the present study is sometimes known as discrete cascade algorithm, which is based on the model described in section 25 of Monin and Yaglom ${ }^{20)}$ and generalized to include Lèvy-stable distribution. 


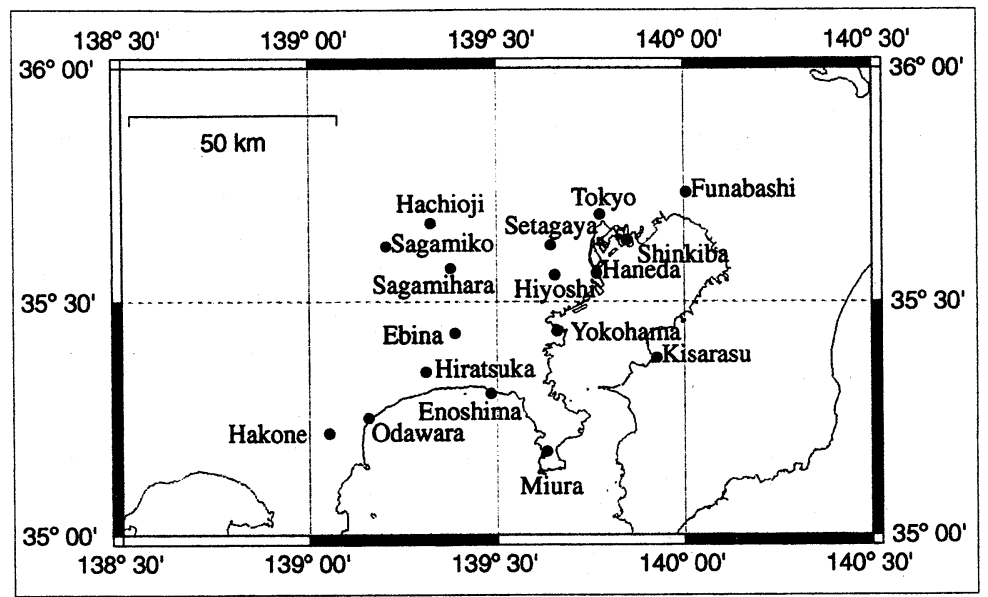

Fig. 2 Location of the rain gauges used for the analysis

Table 1 Properties of the selected rainfall series

\begin{tabular}{lrrrrrr}
\hline & $\begin{array}{r}\text { Elevation } \\
(\mathbf{m})\end{array}$ & $\begin{array}{r}\text { Average Rainfall } \\
(\mathbf{m m} / \mathbf{y r})\end{array}$ & $\begin{array}{r}\text { Record Length } \\
\text { (yrs) }\end{array}$ & $C_{1}$ & $H$ & $\alpha$ \\
\hline Ebina & 18 & 1702 & 22 & 0.35 & -0.06 & 1.34 \\
Enoshima & 60 & 1551 & 17 & 0.32 & -0.04 & 1.35 \\
Funabashi & 24 & 1295 & 20 & 0.32 & -0.05 & 1.37 \\
Hachioji & 121 & 1570 & 8 & 0.40 & -0.07 & 1.09 \\
Hakone & 850 & 3421 & 22 & 0.31 & -0.06 & 1.47 \\
Haneda & 3 & 1374 & 22 & 0.33 & -0.05 & 1.37 \\
Hiratsuka & 20 & 1574 & 22 & 0.35 & -0.06 & 1.45 \\
Hiyoshi & 57 & 1510 & 22 & 0.31 & -0.05 & 1.63 \\
Kisarasu & 5 & 1453 & 22 & 0.35 & -0.05 & 1.21 \\
Miura & 42 & 1567 & 17 & 0.35 & -0.06 & 1.42 \\
Odawara & 28 & 1968 & 22 & 0.39 & -0.05 & 1.09 \\
Sagamihara & 149 & 1677 & 22 & 0.38 & -0.06 & 1.29 \\
Sagamiko & 188 & 1552 & 22 & 0.42 & -0.08 & 1.23 \\
Setagaya & 41 & 1526 & 22 & 0.35 & -0.06 & 1.33 \\
Shinkiba & 6 & 1311 & 22 & 0.34 & -0.05 & 1.31 \\
Tokyo & 7 & 1450 & 22 & 0.36 & -0.05 & 1.19 \\
Yokohama & 39 & 1625 & 22 & 0.26 & -0.04 & 1.92 \\
\hline & & & & $\mathbf{0 . 0 5 6}$ & $\mathbf{1 . 3 5 7}$ \\
& & & $\mathbf{0 . 3 4 5}$ & $\mathbf{- 0 . 0 5}$ \\
\hline
\end{tabular}

The procedure to generate a multifractal field with given multifractal parameters $\left(C_{1}\right.$ and $\left.\alpha\right)$ is as follows: Subdivide a original field of uniform density of unity in to two and multiply each with exponents of an independent Lèvy (stable) random variable. This procedure is reapplied to each part of the resulting distribution, dividing each section of the field in to two equal segments at each step. After a large number of such steps, a field with multifractal properties $C_{1}$ and $\alpha$ can be obtained. For the specific problem discussed in this manuscript, the largest scale of interest $\left(\lambda_{o}\right)$ was selected as 1024 hours, and thus, 10 cascade steps (resulting in 1024 hour series) should be performed. A large number of such distributions give the multifractal field on which the synthetic rainfall series can be based.

\section{(1) From Multifractal Field to Synthetic Rainfall Series}

The discrete cascade algorithm described here does not conserve the mass-balance during cascading process. Thus, the magnitude of the final multifractal field should be adjusted to represent the magnitudes involved in the rainfall series, which is achieved by $R_{g e n}(i)=\mu_{R_{o b s}} M(i) / \mu_{(M)}$, where $M$ is the generated multifractal field, $R_{o b s}$ is the observed daily rainfall field and $R_{g e n}$ is the resulting synthetic rainfall series. Further, the resulting multifractal series do not have zero values, which make it necessary to impose a lower cut-off value to impose the zero values. It is obvious that these two adjustments are dependant of each other, and thus should be performed interactively. However, in this study it was found 
out that, simply cutting off the values below $1 \mathrm{~mm}$ (which is the least measurement of the observed rainfall), after adjusting for the mean does give a reasonable distribution of zero values.

\section{RESULTS}

The resulting synthetic rainfall series were subjected to extensive verification to test the agreement with the observed hourly data. Three levels of verification were performed, namely, 1) the distribution of intensities as a set 2) the ordering properties, by autocorrelation of rainfall time series and 3) properties significant to rainfall series: distribution of rainfall events in duration and magnitude and distribution of zero values.

Fig. 3 shows some scattergrams of rainfall intensities of the hourly synthetic series generated based on daily data and original hourly observations. While the agreement was satisfactory in all of the series analysed, it was found out that the method slightly over estimates the rare-intensities of the synthetic data. At least one past study (Ngugen and Pandey ${ }^{6}$ ) has reported that using of codimension function to describe rainfall intensities, can underestimate the rare events. We find that this discrepancy can largely be attributed to the statistical fitting method used to estimate the $c(\gamma)$ function. Even though it is possible to eliminate such problems by fine-tuning the fitting model, we do not promote such a measure, since, the purpose of this study is to assess the possibility of deriving hourly data when such data is not available and hence no comparison of above nature is possible. However, most of these estimation errors are confined within $10 \%$ error margin.

The autocorrelation structure agrees almost perfectly up to about 10 hours of time lag(Fig. 4). Since, the typical response studies that demand hourly data (e.g. flood response of small urban catchments), have peak-delays of a few hours, this result is very significant. At the long time lag values there definitely is a some small amount of autocorrelation left with the synthetic series, which is not present with the original data. This can be explained in very broad terms: The entire process of modeling rainfall and a multifractal process involves a major simplifying assumption that the rainfall behaves in a certain orderly fashion (i.e. according to a scaling model) The behavior of the natural phenomenon (observed hourly rainfall) may have some 'disorder' (which causes the autocorrelation to become almost zero after about 20 hours) that can not be captured fully by the multifractal model. The 'left-over' autocorrelation may be attributed to the modeling error caused by a 'forced-orderliness', thus imposed by the model.

The 'rainfall events' were identified in the observed and generated rainfall series. In this process we assumed that the occurrence of a dry period of more than $6 \mathrm{hrs}$ separates one rainfall event from the next. The properties of these events are important characteristics of a rainfall series in the viewpoint of short time-scale problems like flood response studies. The duration and the volume of these rainfall event sets were compared for each rain gauge station (Fig. 5 and Fig. 6 ). The event volume shows similar behavior from very short events of frequent occurrence to extremely rare (and long) events. However, for rain event large enough to have more than five years of return period, seems to be overestimated by the multifractal model.

In order to compare the distribution of rainy (and dry) period distribution (i.e. The distribution of zero/non-zero values at each scale) fracttal dimension of rainfall series was calculated. In order to compute the fractal dimension the number of time-steps, $N(l)$ (general term is 'boxes' - for the case of time-series this becomes time-steps) of a given size needed to cover all the non-zero values in the distribution is calculated, for various values of time-steps lengths $(l)$. Fig. 7) shows the distribution of the number of non-zero time$\operatorname{steps}(N(l))$ against the time-steps $\operatorname{size}(l)$. The slope of the straight-line regression of this distribution is defined as the Fractal Dimension of the distribution. The number of 'wet' time-steps is slightly over estimated by the rainfall model at hourly scale (i.e. lesser zero values). However, in many cases the distribution becomes less overestimated (or underestimated in some cases) at coarser time steps. This is due to the fact that almost always the value of fractal dimension is lesser in the generated series than that of the observed series.

\section{CONCLUSIONS}

We propose a complete method to derive synthetic hourly rainfall series from values observed at daily scale. The comparison of numerous properties of those synthetic rainfall series for 17 rain gauge stations is Tokyo area, shows concrete evidence that this method can be practically used for the intended purpose, at least for the rainfall series similar to those used in this study.

Even though the synthetic rainfall series corresponds to their daily counterparts only in a stochastic way (the daily total rainfall is not preserved in a corresponding $24 \mathrm{~h}$ segment of the syn- 

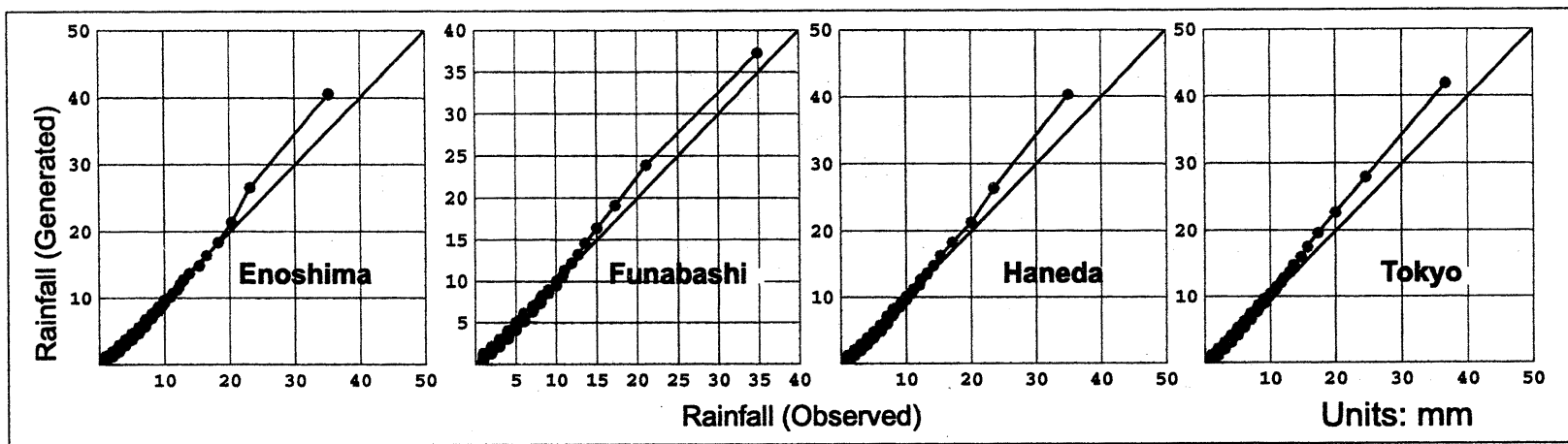

Fig. 3 The comparison of the intensity distribution between observed hourly data and synthetic hourly data. The total set of non-zero values were divided into 400 quintiles in order to make a statistically meaningful comparison. Generated rainfall intensity is almost always slightly over-estimated towards high intensities.

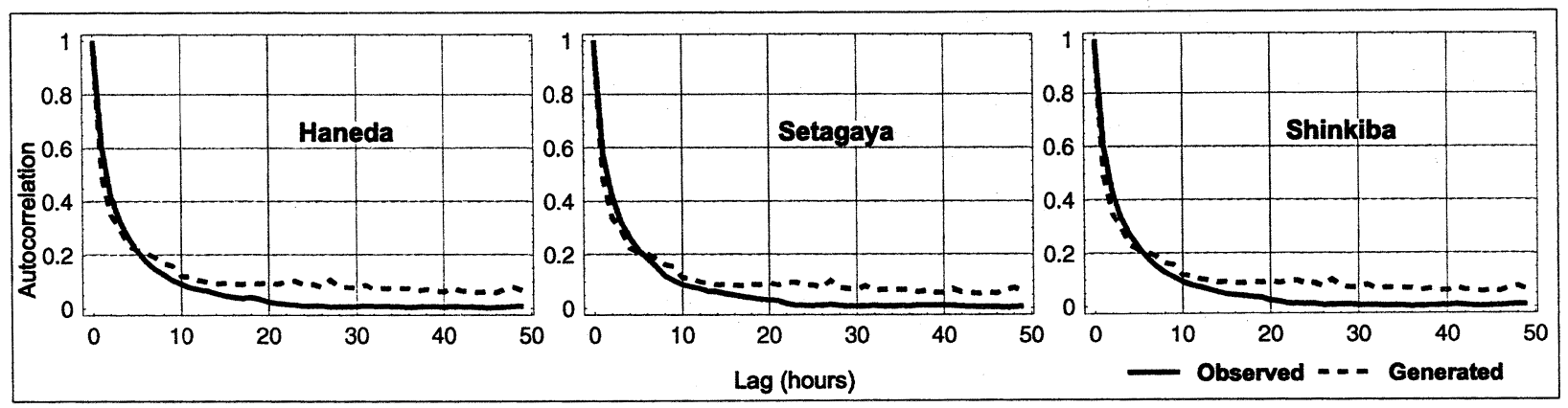

Fig. 4 Autocorrelations of the synthetic series compared with those of original hourly data. As the lag is increased a residual amount is left on the observed series while the original 1hour data shows a continually decaying autocorrelation function.

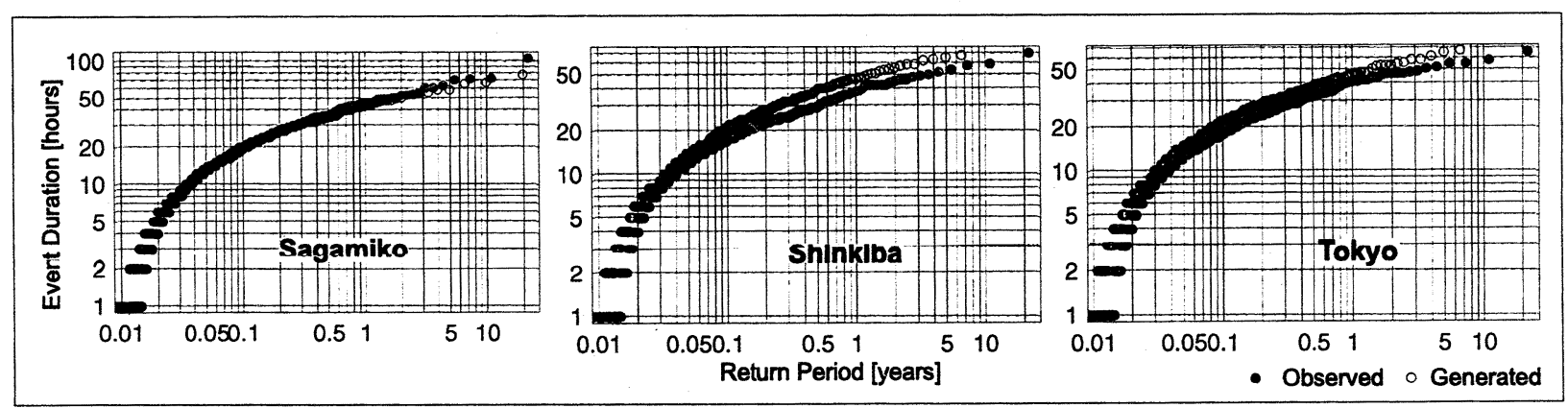

Fig. 5 Comparison of the distribution of rainfall events. Event duration shows a well-behaved variation well into the extreme end. (Minimum dry period separating two event was taken as $6 \mathrm{~h}$ ).

thetic series), the verification show that these results are significantly useful in the real-word hydrological problems like soil erosion studies, urban storm drainage problems and response of small watersheds.

Acknowledgments: We thank J. Olsson of Institute of Environmental Systems (SUIKO) of Kyushu University, Fukuoka and formerly at Department of Water Resources Engineering (TVRL), Lund University, SWEDEN for many helpful comments regarding multifractal analysis. The multifractal simulation model was based on the original discrete cascade algorithm provided by Daniel Lavallèe of the Institute for Crustal Studies University of California, Santa Barbara.

(Received October 2, 2000)

\section{REFERENCES}

1) A. Pathirana, S. Herath and K. Musiake, On the scaling properties of a stochastic rainfall model, Annual Journal of Hydraulic Engineering, JSCE, 44, 2000 February.

2) S. Herath, S. Tanimoto,K. Musiake, K. Hiranoka, A GIS based distributed catchment model for the 

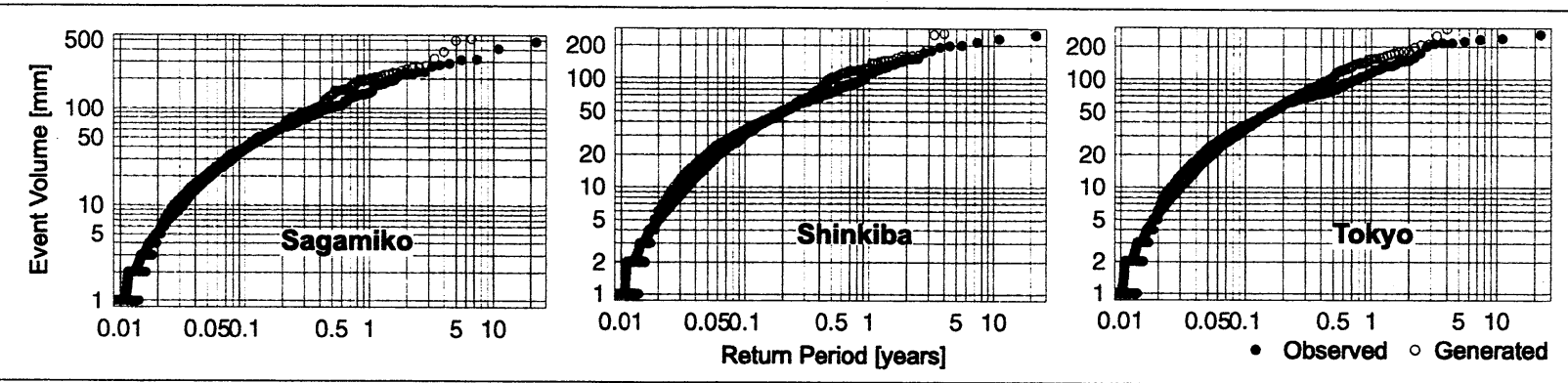

Fig. 6 Event volume discrepancy shows a sudden increase at a extreme value (Minimum dry period separating two event was taken as $6 \mathrm{~h})$.
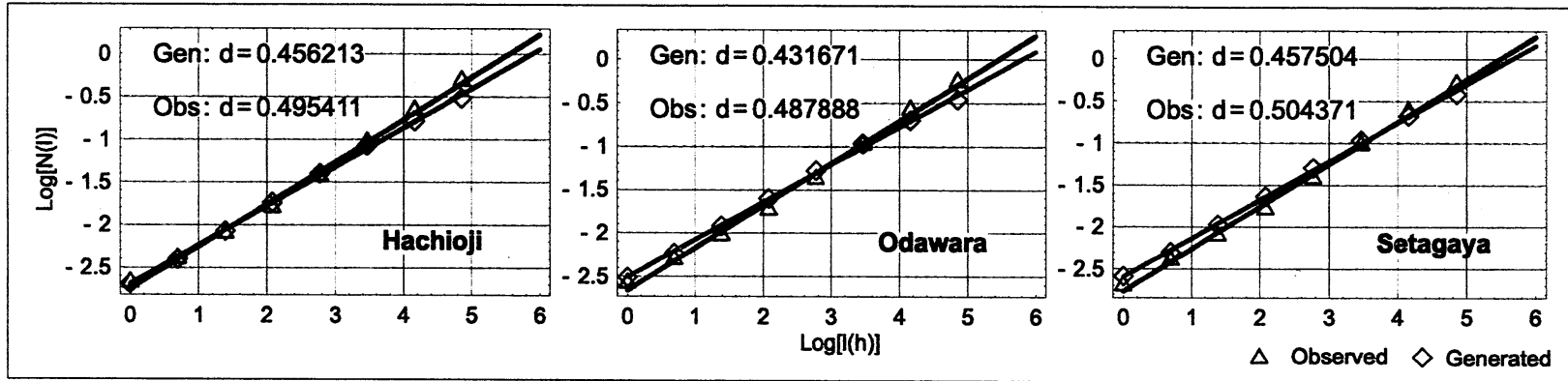

Fig. 7 The distribution of non-zero values in the rainfall series.

simulation of urban hydrology, Proc. 50th Annual Conference of Japan Society of Civil Engineering, July, 2000

3) S. Lovejoy and D. Schertzer, Multifractals, Universality Classes and Satellite and Radar Measurements of Cloud and Rain Fields J. Geophys. Res., 95, 2021-2034, 1990.

4) J. Olsson, Validity and applicability of a scaleindependent multifractal relationship for rainfall Atmospheric Res., 42, 53-65, 1996.

5) C. Svensson, J. Olsson and R. Berndtsson, Multifractal properties of daily rainfall in two different climates Water Resources Res., 32, 8, 2463-2472, 1996.

6) V. Ngugen and G.R. Pandey. Estimation of shortduration rainfall distribution using data measured at longer time scales, Water Science and Tech., 29, 1-1,139-45, 1994.

7) A. Pathirana, S. Herath and K. Musiake, A Multifractal Approach to Predict High-resolution Rinfall Distributions. Proc. 2nd International Symposium of Japan Society of Civil Engineering, July, 2000

8) Y. Tessier, S. Lovejoy and D. Schertzer, Universal Multifractals: theory and observations for rain and clouds, Journal of Appl. Met.,, 2 223-250, 1993

9) E. A. Novikov and R. Stewart, Intermittency of Turbulence and Spectrum of Fluctuations in Energy-Dissipation. Izv. Akad. Nauk. SSSR. Geofiz., 3, 408-412, 1964.

10) A. M. Yaglom, The Influence of the Fluctuation in Energy Dissipation on the Shape of Turbulent Characteristics in the Inertial Interval. Sov. Phys. Dok 2, 26-30, 1966

11) B. Mandelbrot, Intermittent Turbulence in Self-
Similar Cascades: Divergence of High Moments and Dimension of the Carrier. J. Fluid Mech.,62, 331-350, 1974.

12) U. P. Frisch, P. L. Sulem, and M. Nelkin, A Simple Dynamical Model of Intermittency in Fully Developed Turbulence. J. Fluid Mech., 87, 719-724, 1978.

13) R. Benzi , G. Paladin, G. Parisi, and A. Vulpiani, On the Multifractal Nature of Fully Developed Turbulence and Chaotic System. J. Phys., A17, 3521-3531, 1984.

14) C. Meneveau, and K. R. Sreenivasan, Simple Multifractal Cascade Model for Fully Developed Turbulence. Phys. Rev. Lett., 59 (13), 1424-1427, 1987.

15) V. K. Gupta, and E. Waymire, Multiscaling Properties of Spatial Rainfall and River Flow Distributions. J. Geophys. Res., 95, 1999-2010, 1990.

16) D. Schertzer, and S. Lovejoy, Nonlinear variability in Geophysics; Multifractal Simulations and Analysis, in Fractals; Physical Origin and Consequences, Ed. L Pietronero, Plenum, New York, 49-79, 1990.

17) P. Brax, and R. Pechanski, Lèvy Stable Law Description on Intermittent Behavior and Quarkgluon Phase Transitions, Phys. Lett. B, 225-230, 1991.

18) J. Wilson, D. Schertzer and S. Lovejoy Continious Multiplicative Cascade Models of Rain and Clouds, in Non-linear Variability in Geophysics, Kluwer Academic Publishers, pp. 185-207, 1991

19) M. Grigoriu, Applied Non-Gaussian Processes, PTR Prentice Hall, Englewood Cliffs, NJ. 442pp, 1995.

20) A. S. Monin and A. M. Yaglom, Statistical Fluid Mechanics, Vol. 2, The MIT press, 874p., 1975. 